\title{
ISOMORPHISM TYPES OF COMMUTATIVE ALGEBRAS OF FINITE RANK OVER AN ALGEBRAICALLY CLOSED FIELD
}

\author{
BJORN POONEN
}

\begin{abstract}
Let $k$ be an algebraically closed field. We list the finitely many isomorphism types of rank $n$ commutative $k$-algebras for $n \leq 6$. There are infinitely many types for each $n \geq 7$.
\end{abstract}

This article was originally published in Computational Arithmetic Geometry (edited by K. Lauter and K. Ribet), Contemporary Math. 463 (2008), Amer. Math. Soc., 111-120. That version had an error in the classification of symmetric bilinear forms in characteristic 2; this led to a few redundant entries in the table. Also, one algebra in Case 4b in characteristic 2 was missing. These errors have now been corrected.

All algebras are assumed to be commutative, associative, and with 1 (except briefly in Remark 1.1). We assume that $k$ is an algebraically closed field, except in Section 2. By the rank of a $k$-algebra, we mean its dimension as a $k$-vector space.

\section{LOCAL ALGEBRAS OF RANK UP TO 6}

Our main goal is to list representatives for the (finitely many) isomorphism classes of rank $n k$-algebras for $n \leq 6$. As we discuss in Section 2, it is known SSup56 that the number of isomorphism classes is infinite for every $n \geq 7$, so it is natural to stop at 6 . One purpose of these calculations is to give insight into the moduli space of based rank $n$ algebras for small values of $n$ : see [Poo08]. The geometry of this moduli space seems to be what is behind the parameterization and enumeration of number fields of fixed low degree and bounded discriminant, as in the work of Bhargava Bha04a, Bha04b, Bha04c, Bha05].

Remark 1.1. Many partial results had been obtained by earlier authors. For example, in the case char $k=0$, these algebras were determined implicitly, by classifying nilpotent commutative subalgebras of the algebra of $n \times n$ matrices up to conjugacy, by Cha54 $(n \leq 5)$ and [Dym66] $(n=6)$. See also [ST66, §2.8 and §2.9]. The paper [Maz80] classifies nilpotent commutative associative algebras without 1 up to $n \leq 5$ for char $k \neq 2,3$; isomorphism types of such algebras of rank $n$ are in bijection with isomorphism types of local commutative associative algebras with 1 of rank $n+1$ (send $A$ to $k \oplus A$ ), so our results are novel only in that they handle the cases of characteristic 2 and 3. Our methods are more elementary than those in [Maz80, which used Hochschild cocycles.

Remark 1.2. The classification of finite-rank algebras is useful in the study of regular subgroups of the affine group $\mathrm{AGL}_{n}(k)$; see PTB16].

Date: November 16, 2007 (corrections made December 4, 2020).

2000 Mathematics Subject Classification. Primary 13E10.

Key words and phrases. Artinian, finite-dimensional algebras. 
Classifying the local algebras $A$ is enough since every finite-rank algebra is a product of these. Let $\mathfrak{m}$ be the maximal ideal of $A$. For $i \geq 1$, let $d_{i}=\operatorname{dim}_{k}\left(\mathfrak{m}^{i} / \mathfrak{m}^{i+1}\right)$.

We classify local algebras $A$ first by $n:=\operatorname{dim}_{k} A$, and next by the sequence $\vec{d}:=\left(d_{i}\right)_{i \geq 1}$ (we retain only the finitely many nonzero terms). Each algebra is represented by $P / I$ where $P$ is the polynomial ring in the first $d_{1}$ of the variables $x, y, z, w, v$, and $I$ is an ideal in $P$. Table 1 summarizes the results. For each $(n, \vec{d})$, it lists exactly one algebra in each isomorphism class, by giving $I$. Each entry marked with * (or **) should be included if and only if char $k=2$ (respectively, 3): in other characteristics it becomes isomorphic to the last unstarred entry preceding it.

\section{Lemma 1.3.}

(1) If $d_{1}=1$, then $A \simeq k[x] /\left(x^{n}\right)$.

(2) If $d_{1}=n-1$, then $A \simeq k\left[x_{1}, \ldots, x_{n-1}\right] /\left(x_{1}, \ldots, x_{n-1}\right)^{2}$.

(3) The local algebras with $\vec{d}=\left(d_{1}, d_{2}\right)$ correspond to $d_{2}$-dimensional subspaces of the space of symmetric bilinear forms on a $d_{1}$-dimensional vector space $V$, up to $\mathrm{GL}(V)$ equivalence. Equivalently, they correspond to $\left(d_{1}\left(d_{1}+1\right) / 2-d_{2}\right)$-dimensional subspaces of the space of quadratic forms on a $d_{1}$-dimensional vector space $W$, up to $\mathrm{GL}(W)$-equivalence.

Proof. The first two claims are obvious. For the third statement we take $V:=\mathfrak{m} / \mathfrak{m}^{2}$ and consider the subspace of bilinear forms obtained by composing the multiplication map $V \times V \rightarrow \mathfrak{m}^{2}$ with linear functionals $\mathfrak{m}^{2} \rightarrow k$. Taking the dual of the subspace gives a space of quadratic forms on $W:=V^{\vee}$.

Remark 1.4 (Classification of symmetric bilinear forms). Symmetric bilinear forms correspond to symmetric matrices. We can form such a symmetric matrix by taking the direct sum of a zero block and an identity matrix block. If char $k=2$, we can also take the direct sum of a zero block and blocks of the form $H:=\left(\begin{array}{ll}0 & 1 \\ 1 & 0\end{array}\right)$. Albert Alb38, Theorems 3 and 6] proved that every symmetric matrix is equivalent to a unique matrix in the list just given. (Thus for $n \geq 1$, the number of types of nondegenerate symmetric bilinear forms on an $n$-dimensional space is two if char $k=2$ and $n$ is even, and one if char $k \neq 2$ or $n$ is odd.) We use the notation $0_{a} \oplus 1_{b}$ to denote the direct sum of an $a \times a$ zero block and a $b \times b$ identity matrix block.

Example 1.5. Rank 5 algebras with $\vec{d}=(3,1)$ are given by a nonzero symmetric bilinear form on a 3-dimensional space, up to equivalence. Such an algebra has a basis $1, e_{2}, e_{3}, e_{4}, e_{5}$ and the entries of the $3 \times 3$ symmetric matrix specify what multiple of $e_{5}$ we get when we multiply two of $e_{2}, e_{3}, e_{4}$. For example, if in characteristic 2 we choose the type that is a direct sum of $(0)$ and $\left(\begin{array}{ll}0 & 1 \\ 1 & 0\end{array}\right)$, we get $k[x, y, z]$ modulo the ideal sum of $(x, y, z)^{3}$ and $\left(x^{2}, y^{2}, z^{2}, x y, x z\right)$ (coming from the zeros in the $3 \times 3$ matrix); this is the second algebra in Table 1 with $\vec{d}=(3,1)$; in the explanation column, we give the shorthand $0_{1} \oplus H$ for the type of the symmetric bilinear form.

Remark 1.6 (Classification of quadratic forms). Every quadratic form in $x_{1}, \ldots, x_{n}$ is equivalent to $x_{1} x_{2}+x_{3} x_{4}+\cdots+x_{2 r-1} x_{2 r}$ or $\left(x_{1} x_{2}+x_{3} x_{4}+\cdots+x_{2 r-1} x_{2 r}\right)+x_{2 r+1}^{2}$ for some $r \in \mathbb{Z}_{\geq 0}$. 


\begin{tabular}{|c|c|c|c|}
\hline$n$ & $\vec{d}$ & Ideal & Explanation \\
\hline 1 & & $(0)$ & \\
\hline 2 & 1 & $\left(x^{2}\right)$ & $\begin{array}{ll}\text { Lemma } 1.3(1) \\
\end{array}$ \\
\hline & 1,1 & $\left(x^{3}\right)$ & Lemma $1.3(1)$ \\
\hline & 2 & $(x, y)^{2}$ & Lemma $1.3(2)$ \\
\hline \multirow[t]{5}{*}{4} & $1,1,1$ & $\left(x^{4}\right)$ & Lemma $1.3(1)$ \\
\hline & 2,1 & $\left(x^{2}, x y, y^{3}\right)$ & $0_{1} \oplus 1_{1}$ \\
\hline & & $\left(x^{2}, y^{2}\right)$ & $H$ \\
\hline & & $*\left(x^{2}+y^{2}, x y\right)$ & $1_{2}$ \\
\hline & 3 & $(x, y, z)^{2}$ & Lemma $1.3(2)$ \\
\hline \multirow[t]{9}{*}{5} & $1,1,1,1$ & $\left(x^{5}\right)$ & Lemma $1.3(1)$ \\
\hline & $2,1,1$ & $\begin{array}{c}\left(x^{2}, x y, y^{4}\right) \\
\left(x^{2}+y^{3}, x y\right)\end{array}$ & \\
\hline & 2,2 & $\left(x y, x^{3}, y^{3}\right)$ & Lemma $1.3(3)$, Remark 1.6 \\
\hline & & $\left(x^{2}, x y^{2}, y^{3}\right)$ & Lemma $1.3(3)$, Remark 1.6 \\
\hline & 3,1 & $\left(x^{2}, y^{2}, x y, x z, y z, z^{3}\right)$ & $0_{2}$ \\
\hline & & $\left(x^{2}, y^{2}, z^{2}, x y, x z\right)$ & $0_{1} \oplus H$ \\
\hline & & $*\left(x^{2}, x y, x z, y z, y^{2}+z^{2}\right)$ & $0_{1} \oplus 1_{2}$ \\
\hline & & $\left(x y, x z, y z, x^{2}+y^{2}, x^{2}+z^{2}\right)$ & $1_{3}$ \\
\hline & 4 & $(x, y, z, w)^{2}$ & Lemma $1.3(2)$ \\
\hline \multirow[t]{34}{*}{6} & $1,1,1,1,1$ & $\left(x^{6}\right)$ & Lemma $1.3(1)$ \\
\hline & $2,1,1,1$ & $\left(x^{2}, x y, y^{5}\right)$ & \\
\hline & & $\left(x^{2}+y^{4}, x y\right)$ & \\
\hline & $2,2,1$ & $\left(x y, x^{3}, y^{4}\right)$ & Case I \\
\hline & & $\left(x y, x^{3}+y^{3}\right)$ & Case I \\
\hline & & $\left(x^{2}, x y^{2}, y^{4}\right)$ & Case IIa \\
\hline & & $\left(x^{2}+y^{3}, x y^{2}, y^{4}\right)$ & Case IIa \\
\hline & & $\left(x^{2}, y^{3}\right)$ & Case IIa \\
\hline & & $*\left(x^{2}+x y^{2}, y^{3}\right)$ & Case IIa \\
\hline & & $* *\left(x^{2}, x y^{2}+y^{3}\right)$ & Case IIb \\
\hline & 2,3 & $(x, y)^{3}$ & Lemma $1.3(3)$ \\
\hline & $3,1,1$ & $\left(x^{2}, x y, y^{2}, x z, y z, z^{4}\right)$ & $0_{2}$ \\
\hline & & $\left(x^{2}, x y, y^{2}+z^{3}, x z, y z, z^{4}\right)$ & $0_{1} \oplus 1_{1}$ \\
\hline & & $\left(x^{2}, x y+z^{3}, y^{2}, x z, y z, z^{4}\right)$ & $H$ \\
\hline & & $*\left(x^{2}+z^{3}, x y, y^{2}+z^{3}, x z, y z, z^{4}\right)$ & $1_{2}$ \\
\hline & 3,2 & $\left(x y, y z, z^{2}, y^{2}-x z, x^{3}\right)$ & Case 1a \\
\hline & & $\left(x y, z^{2}, x z-y z, x^{2}+y^{2}-x z\right)$ & Case $1 b$ \\
\hline & & $*\left(x^{2}, z^{2}, y^{2}-x z, y z\right)$ & Case $1 c$ \\
\hline & & $\left(x^{2}, x y, x z, y^{2}, y z^{2}, z^{3}\right)$ & Case 2 \\
\hline & & $\left(x^{2}, x y, x z, y z, y^{3}, z^{3}\right)$ & Case 2 \\
\hline & & $\left(x y, x z, y^{2}, z^{2}, x^{3}\right)$ & Case 3 \\
\hline & & $*\left(x y, x z, y z, y^{2}-z^{2}, x^{3}\right)$ & Case 3 \\
\hline & & $\left(x y, x z, y z, x^{2}+y^{2}-z^{2}\right)$ & Case $4 \mathrm{a}$ \\
\hline & & $\left(x^{2}, x y, y z, y^{2}-z^{2}\right)$ & Case $4 \mathrm{~b}$ \\
\hline & & $*\left(x^{2}, x y, y z, x z+y^{2}-z^{2}\right)$ & Case $4 \mathrm{~b}$ \\
\hline & & $\left(x^{2}, x y, y^{2}, z^{2}\right)$ & Case $4 \mathrm{~d}$ \\
\hline & & $*\left(x^{2}, x y, y^{2}, z^{2}-x z\right)$ & Case $4 \mathrm{~d}$ \\
\hline & 4,1 & $\left(x^{2}, y^{2}, z^{2}, x y, x z, x w, y z, y w, z w, w^{3}\right)$ & $0_{3} \oplus 1_{1}$ \\
\hline & & $\left(x^{2}, y^{2}, z^{2}, w^{2}, x y, x z, x w, y z, y w\right)$ & $0_{2} \oplus H$ \\
\hline & & $*\left(x^{2}, y^{2}, z^{2}+w^{2}, x y, x z, x w, y z, y w, z w\right)$ & $0_{2} \oplus 1_{2}$ \\
\hline & & $\left(x^{2}, y^{2}+z^{2}, y^{2}+w^{2}, x y, x z, x w, y z, y w, z w\right)$ & $0_{1} \oplus 1_{3}$ \\
\hline & & $\left(x^{2}, y^{2}, z^{2}, w^{2}, x y-z w, x z, x w, y z, y w\right)$ & $H \oplus H$ \\
\hline & 5 & $\begin{array}{c}*\left(x^{2}+y^{2}, x^{2}+z^{2}, x^{2}+w^{2}, x y, x z, x w, y z, y w, z w\right) \\
(x, y, z, w, v)^{2}\end{array}$ & $\begin{array}{c}1_{4} \\
\text { Lemma } \\
1.3(2)\end{array}$ \\
\hline & & & \\
\hline
\end{tabular}

TABLE 1. Local algebras over $k=\bar{k}$ of rank $\leq 6$. 
Lemma 1.3 and Remarks 1.4 and 1.6 immediately classify all algebras of rank $\leq 6$ except for those with $n$ and $\vec{d}$ as follows:

$$
\begin{aligned}
& n=5:(2,1,1) \\
& n=6:(2,1,1,1),(2,1,2),(2,2,1),(3,1,1),(3,2) .
\end{aligned}
$$

So we now suppose that $A$ is an algebra of one of these types. Recall that we write $A=P / I$.

\section{1. $\vec{d}=(2,1,1)$ or $\vec{d}=(2,1,2)$. Then $A / \mathfrak{m}^{3}$ is a rank 4 algebra with $\vec{d}=(2,1)$.}

Suppose $A / \mathfrak{m}^{3}$ is isomorphic to $k[x, y] /\left(x^{2}, y^{2}\right)$ or $k[x, y] /\left(x^{2}+y^{2}, x y\right)$. Then the ideal $I$ contains two polynomials whose lowest-degree homogeneous parts are of degree 2 and have no common factor. But then $\operatorname{dim}_{k} A$ is at most their intersection multiplicity 4, a contradiction.

Suppose $A / \mathfrak{m}^{3} \simeq k[x, y] /\left(x^{2}, x y, y^{3}\right)$. So $I$ contains $(x, y)^{4}$ as well as $x^{2}+f$ and $x y+g$ for some homogeneous cubic forms $f$ and $g$ in $x, y$. By performing the change of variable $(x, y) \mapsto\left(x+c y^{2}, y\right)$ for some $c \in k$ (this preserves $\left(x^{2}, x y, y^{3}\right)$ ), we may assume that the coefficient of $y^{3}$ in $g$ is 0 . Multiplying $x^{2}+f(x, y), x y+g(x, y)$ by $x, y$ and reducing modulo $(x, y)^{4}$ shows that $x^{3}, x^{2} y, x y^{2} \in I$, so we may assume $f=c y^{3}$ for some $c \in k$ and $g=0$. Scaling $y$, we may assume $c \in\{0,1\}$. Thus $I$ contains either $\left(x^{2}, x y, y^{4}\right)$ or $\left(x^{2}+y^{3}, x y\right)$. But each of these has codimension 5 in $k[x, y]$, so $I$ equals one of these. In particular, algebras with $\vec{d}=(2,1,2)$ do not exist. These two $I$ 's lead to non-isomorphic algebras, since $\operatorname{dim}_{k}(\operatorname{Ann} \mathfrak{m})$ is 2 in the first case, and 1 in the second.

1.2. $\vec{d}=(2,1,1,1)$. Then $A / \mathfrak{m}^{4}$ is isomorphic to $k[x, y] /\left(x^{2}, x y, y^{4}\right)$ or $k[x, y] /\left(x^{2}+y^{3}, x y\right)$.

In the first case, a calculation similar to the case $A / \mathfrak{m}^{3} \simeq k[x, y] /\left(x^{2}, x y, y^{3}\right)$ above shows that $A$ is isomorphic to one of $k[x, y] /\left(x^{2}, x y, y^{5}\right)$ or $k[x, y] /\left(x^{2}+y^{4}, x y\right)$, and that these are non-isomorphic.

In the second case, $I$ contains $(x, y)^{5}$ as well as $x^{2}+y^{3}+f$ and $x y+g$ for some homogeneous quartic forms $f, g$. Multiplying $x^{2}+y^{3}+f$ and $x y+g$ by monomials of degree 2 yields $x^{4}, x^{3} y, x^{2} y^{2}, x y^{3} \in I$. Considering $y\left(x^{2}+y^{3}+f\right)-x(x y+g)$ modulo $(x, y)^{5}$ shows that $y^{4} \in I$. Thus $(x, y)^{4} \subseteq I$, contradicting $d_{4}=1$.

1.3. $\vec{d}=(2,2,1)$. Then $A / \mathfrak{m}^{3}$ is isomorphic to $k[x, y] /\left(x y, x^{3}, y^{3}\right)$ or $k[x, y] /\left(x^{2}, x y^{2}, y^{3}\right)$.

Case I: We have $A / \mathfrak{m}^{3} \simeq k[x, y] /\left(x y, x^{3}, y^{3}\right)$. Then $I$ contains $(x, y)^{4}$ as well as $x y+f$ for some cubic form $f$, and after changes of variables $(x, y) \mapsto\left(x, y+x^{2}\right)$ and $(x, y) \mapsto\left(x+y^{2}, y\right)$ we may assume that $x^{3}$ and $y^{3}$ do not occur in $f$. Considering $x(x y+f), y(x y+f)$ modulo $(x, y)^{4}$ yields $x^{2} y, x y^{2} \in I$, so we may assume $f=0$. Now $(x y)+(x, y)^{4}$ has codimension 7 , so $I=(x y, g)+(x, y)^{4}$ for some nonzero cubic form $g$, which we may take to be of the form $a x^{3}+b y^{3}$. By interchanging and scaling $x, y$, we may assume $g=x^{3}$ or $g=x^{3}+y^{3}$. These give non-isomorphic algebras, $\operatorname{since} \operatorname{dim}_{k}($ Ann $\mathfrak{m})$ is 2 or 1 , respectively.

Case II: We have $A / \mathfrak{m}^{3} \simeq k[x, y] /\left(x^{2}, x y^{2}, y^{3}\right)$. Then $I$ contains $(x, y)^{4}$ as well as $x^{2}+f$ for some cubic form $f$. Considering $x\left(x^{2}+f\right), y\left(x^{2}+f\right)$ modulo $(x, y)^{4}$ yields $x^{3}, x^{2} y \in I$. But $(x, y)^{4}+\left(x^{2}+f\right)$ has codimension 7 , so $I$ contains also a nonzero cubic form $g=c x y^{2}+d y^{3}$. The cubic form $g$ is determined up to scalar multiple and up to performing a change of variable $(x, y) \mapsto\left(\lambda x, \mu x+\mu^{\prime} y\right)$ and reducing modulo the span of $x^{3}$ and $x^{2} y$, since these are the only linear changes of variable preserving $\left(x^{2}, x y^{2}, y^{3}\right)$.

Case IIa: We can make $g$ so that $c=0$ or $d=0$; by the previous sentence, this condition is determined by the isomorphism type of $A$. Then scaling $x, y$ and scaling $g$, we may assume 
$g=x y^{2}$ or $g=y^{3}$. Now reducing $f$ modulo $\left(x^{3}, x^{2} y, g\right)$ and scaling, we may assume that if $g=x y^{2}$ then $f=0$ or $f=y^{3}$, and if $g=y^{3}$ then $f=0$ or $f=x y^{2}$. Thus we have four possibilities for $I$ :

$$
\left(x^{2}, x y^{2}, y^{4}\right),\left(x^{2}+y^{3}, x y^{2}, y^{4}\right),\left(x^{2}, y^{3}\right),\left(x^{2}+x y^{2}, y^{3}\right) .
$$

We claim that these are non-isomorphic, except that the last two are isomorphic if char $k \neq 2$ via $(x, y) \mapsto\left(x+y^{2} / 2, y\right)$. We have $\operatorname{dim}(\operatorname{Ann} \mathfrak{m})=2,2,1,1$, respectively. The first is distinguished from the second (and the third from the fourth if char $k=2$ ) by the existence of an element of $\mathfrak{m}-\mathfrak{m}^{2}$ whose square is 0 .

Case IIb: We cannot make $c=0$ or $d=0$. This can happen only if char $k=3$, since otherwise when $d \neq 0$ a linear change of variable $(x, y) \mapsto(x, y+\alpha x)$ can make $c=0$. Scaling $x$ and $y$, we may assume that $g=x y^{2}+y^{3}$. Adding a multiple of $g$ to $x^{2}+f \in I$, we may assume that $f=a x y^{2}$ for some $a \in k$. If we use the change of variable $(x, y) \mapsto\left(x-a y^{2} / 2, y\right)$ and reduce modulo $(x, y)^{4}+\left(x^{3}, x^{2} y\right)$, we may assume $a=0$ without changing $g$. This leads to the algebra $k[x, y] /\left(x^{2}, x y^{2}+y^{3}\right)$. We check that when char $k=3$, no change of variable as mentioned before could have made $c=0$ or $d=0$, and hence this algebra is not isomorphic to those in Case IIa. On the other hand, when char $k \neq 3$, it is isomorphic to $k[x, y] /\left(x^{2}, y^{3}\right)$ via $(x, y) \mapsto(x, y+x / 3)$.

1.4. $\vec{d}=(3,1,1)$. Then $A / \mathfrak{m}^{3}$ is one of the four algebras with $\vec{d}=(3,1)$.

Suppose $A / \mathfrak{m}^{3} \simeq k[x, y, z] /\left(x^{2}, y^{2}, x y, x z, y z, z^{3}\right)$. Then $I$ contains $x^{2}+f_{1}, y^{2}+f_{2}, x y+$ $f_{3}, x z+f_{4}, y z+f_{5}$ and $(x, y, z)^{4}$ for some cubic forms $f_{1}, \ldots, f_{5}$. Multiplying by variables and reducing modulo $(x, y, z)^{4}$ shows that all cubic monomials are in $I$ except possibly $z^{3}$, so $z^{3}$ is a basis for $\mathfrak{m}^{3}$. So we may assume $f_{i}=c_{i} z^{3}$ for some $c_{i} \in k$. Replacing $x$ by $x+a z^{2}$ and $y$ by $y+b z^{2}$ for suitable $a, b \in k$, we may assume that $f_{4}=f_{5}=0$. Thus $A$ is determined by $c_{1}, c_{2}, c_{3}$, which correspond to a symmetric bilinear form from the span $\langle x, y\rangle$ to $\left\langle z^{3}\right\rangle$, or more canonically from $\left(\mathfrak{m}^{3}: \mathfrak{m}\right) / \mathfrak{m}^{2}$ to $\mathfrak{m}^{3}$. The isomorphism type of $A$ is then uniquely determined by the isomorphism type of this symmetric bilinear form. For example, the form given by $\left(\begin{array}{ll}0 & 1 \\ 1 & 0\end{array}\right)$ corresponds to $I=\left(x^{2}, x y-z^{3}, y^{2}, x z, y z, z^{4}\right)$. In summary (changing the sign of $z$ ), we get the four possibilities listed for $\vec{d}=(3,1,1)$.

Suppose $A / \mathfrak{m}^{3} \simeq k[x, y, z] /\left(x^{2}, y^{2}, z^{2}, x y, x z\right)$. As in the previous paragraph, $I$ contains $x^{2}+f_{1}, \ldots, x z+f_{5}$ for some cubic forms $f_{i}$, but this time every cubic monomial is a multiple of one of $x^{2}, y^{2}, z^{2}, x y, x z$, so we can prove that $\mathfrak{m}^{3}=0$, a contradiction.

The other two possibilities for $A / \mathfrak{m}^{3}$ can likewise be ruled out: in each, the multiples of the quadratic monomials in the ideal of $A / \mathfrak{m}^{3}$ span the space of cubic forms.

1.5. $\vec{d}=(3,2)$. By Lemma $1.3, A$ is determined by a 2-dimensional subspace of the space of symmetric bilinear forms on $\langle x, y, z\rangle$, up to $\mathrm{GL}_{3}$-equivalence. Hence $A$ may be represented by a linearly independent pair of symmetric bilinear forms $\left(\langle,\rangle_{1},\langle,\rangle_{2}\right)$ which in turn may be represented by a pair of $3 \times 3$ symmetric matrices $\left(M_{1}, M_{2}\right)$. Let $\operatorname{ker}_{i}$ be the kernel of $\langle,\rangle_{i}$. Let $F(t, u):=\operatorname{det}\left(t M_{1}+u M_{2}\right)$, which is a cubic form determined up to $\mathrm{GL}_{2}$-equivalence by $A$.

Case 1: $F(t, u)$ is a nonzero cube. Without loss of generality, assume that $F(t, u)$ is a multiple of $u^{3}$. In particular det $M_{1}=0$. By Remark 1.4. we may change the basis of $\langle x, y, z\rangle$ 
to assume that $M_{1}$ is one of the following:

$$
\left(\begin{array}{lll}
1 & 0 & 0 \\
0 & 0 & 0 \\
0 & 0 & 0
\end{array}\right),\left(\begin{array}{ccc}
1 & 0 & 0 \\
0 & -1 & 0 \\
0 & 0 & 0
\end{array}\right), \quad\left(\begin{array}{lll}
0 & 1 & 0 \\
1 & 0 & 0 \\
0 & 0 & 0
\end{array}\right)
$$

where the last is needed only if $\operatorname{char} k=2$. Write $M_{2}=\left(\begin{array}{lll}a & b & c \\ b & d & e \\ c & e & f\end{array}\right)$.

Case 1a: $M_{1}=\left(\begin{array}{lll}1 & 0 & 0 \\ 0 & 0 & 0 \\ 0 & 0 & 0\end{array}\right)$. Since $F(t, u)$ is a nonzero multiple of $u^{3}$, the lower right $2 \times 2$ block of $M_{2}$, representing $\left.\langle,\rangle_{2}\right|_{\text {ker }_{1}}$, is a nonzero singular matrix, and by Remark 1.4 a linear change of variables of $y, z$ transforms this block to $\left(\begin{array}{ll}1 & 0 \\ 0 & 0\end{array}\right)$. By replacing $x$ with $x+\lambda y$ for suitable $y$, we may assume $b=0$. By adding a multiple of $M_{1}$ to $M_{2}$ we may assume $a=0$. Now $M_{2}=\left(\begin{array}{lll}0 & 0 & c \\ 0 & 1 & 0 \\ c & 0 & 0\end{array}\right)$. Since $\operatorname{det} M_{2} \neq 0$, we may scale $z$ to assume $c=1$. This $\left(M_{1}, M_{2}\right)$ yields the algebra

$$
A \simeq \frac{k[x, y, z]}{\left(x y, y z, z^{2}, y^{2}-x z\right)+(x, y, z)^{3}} .
$$

Case 1b: $M_{1}=\left(\begin{array}{ccc}1 & 0 & 0 \\ 0 & -1 & 0 \\ 0 & 0 & 0\end{array}\right)$. Adding a multiple of $M_{1}$ to $M_{2}$, we may assume that $d=0$. Setting the coefficients of $t^{2} u$ and $t u^{2}$ in $F(t, u)=\operatorname{det}\left(t M_{1}+u M_{2}\right)$ equal to 0 yields $f=0$ and $c= \pm e$. Moreover, $F(t, u)$ is not identically 0 , so $(c, e) \neq(0,0)$. If necessary, the change of variable $x \mapsto-x$ lets us assume that $c=e$. The change of variable $x \mapsto x+\lambda z$ for suitable $\lambda \in k$ lets us assume in addition that $b=0$. Since $F(t, u)$ is not $0, a \neq 0$. Scaling $M_{2}$, we may assume $a=1$. Scaling $z$, we may assume $c=e=1$. Now $M_{2}=\left(\begin{array}{lll}1 & 0 & 1 \\ 0 & 0 & 1 \\ 1 & 1 & 0\end{array}\right)$, and

$$
A \simeq \frac{k[x, y, z]}{\left(x y, z^{2}, x z-y z, x^{2}+y^{2}-x z\right)+(x, y, z)^{3}} .
$$

Case 1c: $M_{1}=\left(\begin{array}{lll}0 & 1 & 0 \\ 1 & 0 & 0 \\ 0 & 0 & 0\end{array}\right)$ and char $k=2$. Adding a multiple of $M_{1}$ to $M_{2}$, we may assume $b=0$. Since $F$ is a multiple of $u^{3}$, we have $f=0$. Since $M_{2}$ is nonsingular, either $a$ or $d$ is nonzero. By performing a $2 \times 2$ invertible transformation of $x$ and $y$ (and then scaling $M_{1}$ to restore it) we may assume that $a=0$ and $d=1$. Since $M_{2}$ is nonsingular, $c \neq 0$. A change of variable $y \mapsto y+\lambda x$ lets us assume that $e=0$. Scaling $z$, we may assume $c=1$. Now

$$
M_{2}=\left(\begin{array}{lll}
0 & 0 & 1 \\
0 & 1 & 0 \\
1 & 0 & 0
\end{array}\right),
$$


and

$$
A \simeq \frac{k[x, y, z]}{\left(x^{2}, z^{2}, y^{2}-x z, y z\right)+(x, y, z)^{3}}
$$

This completes Case 1.

If $F(t, u)$ is not a nonzero cube, then it vanishes at at least two $(t: u) \in \mathbb{P}^{1}(k)$, so without loss of generality $\mathrm{ker}_{1}$ and $\mathrm{ker}_{2}$ are nonzero.

Case 2: $\operatorname{ker}_{1} \cap \operatorname{ker}_{2} \neq 0$. In this case $\operatorname{dim}\left(\operatorname{ker}_{1} \cap \operatorname{ker}_{2}\right)=1$, since if it were any larger, then $M_{1}$ and $M_{2}$ would be contained in a 1-dimensional space. Without loss of generality, $\operatorname{ker}_{1} \cap \operatorname{ker}_{2}=\langle x\rangle$. Then

$$
A \simeq \frac{k[x, y, z]}{\left(x^{2}, x y, x z, q\right)+(x, y, z)^{3}}
$$

where $q$ is a quadratic form in $y, z$; up to isomorphism there are only the possibilities $q=y^{2}$ and $q=y z$.

Case 3: ker $_{1}$ and ker $_{2}$ have dimensions 1,2, respectively, and do not intersect. Without loss of generality, $\operatorname{ker}_{1}=\langle x\rangle$ and $\operatorname{ker}_{2}=\langle y, z\rangle$. The restriction of each symmetric bilinear form to the kernel of the other is nondegenerate (because of the kernel dimensions), so up to isomorphism Remark 1.4 leaves only the possibility

$$
M_{1}=\left(\begin{array}{ccc}
0 & 0 & 0 \\
0 & 0 & 1 \\
0 & 1 & 0
\end{array}\right), \quad M_{2}=\left(\begin{array}{ccc}
1 & 0 & 0 \\
0 & 0 & 0 \\
0 & 0 & 0
\end{array}\right), \quad A \simeq \frac{k[x, y, z]}{\left(x y, x z, y^{2}, z^{2}\right)+(x, y, z)^{3}},
$$

except that in characteristic 2 we have also

$$
M_{1}=\left(\begin{array}{ccc}
0 & 0 & 0 \\
0 & 1 & 0 \\
0 & 0 & 1
\end{array}\right), \quad M_{2}=\left(\begin{array}{ccc}
1 & 0 & 0 \\
0 & 0 & 0 \\
0 & 0 & 0
\end{array}\right), \quad A \simeq \frac{k[x, y, z]}{\left(x y, x z, y z, y^{2}-z^{2}\right)+(x, y, z)^{3}}
$$

Case 4: $\operatorname{ker}_{1}$ and $\mathrm{ker}_{2}$ are 1-dimensional and do not intersect. Without loss of generality, $\operatorname{ker}_{1}=\langle x\rangle$ and $\operatorname{ker}_{2}=\langle y\rangle$. Then

$$
M_{1}=\left(\begin{array}{lll}
0 & 0 & 0 \\
0 & a & b \\
0 & b & c
\end{array}\right), \quad M_{2}=\left(\begin{array}{lll}
d & 0 & e \\
0 & 0 & 0 \\
e & 0 & f
\end{array}\right)
$$

for some $a, b, c, d, e, f \in k$.

Case 4a: $a, d \neq 0$. By a change of variable $z \mapsto z+\lambda y$ for suitable $\lambda \in k$, we may assume $b=0$. Similarly, by a change $z \mapsto z+\lambda^{\prime} x$, we may assume $e=0$. Since $\operatorname{dim} \operatorname{ker}_{1}=\operatorname{dim} \operatorname{ker}_{2}=$ 1 , we have $c, f \neq 0$. Scaling the $M_{i}$ we may assume $c=f=1$. Scaling $y$ we may assume $a=1$. Scaling $x$ we may assume $d=1$. Now

$$
M_{1}=\left(\begin{array}{lll}
0 & 0 & 0 \\
0 & 1 & 0 \\
0 & 0 & 1
\end{array}\right), \quad M_{2}=\left(\begin{array}{ccc}
1 & 0 & 0 \\
0 & 0 & 0 \\
0 & 0 & 1
\end{array}\right), \quad A \simeq \frac{k[x, y, z]}{\left(x y, x z, y z, x^{2}+y^{2}-z^{2}\right)+(x, y, z)^{3}}
$$

Case 4b: $a \neq 0$ and $d=0$. As in Case 4a, we may assume $b=0$. Since $\operatorname{dim}_{k_{1}}=$ $\operatorname{dim} \operatorname{ker}_{2}=1$, we have $c, e \neq 0$. Scaling, we may assume $a=c=e=1$ and $f \in\{0,1\}$. These 
two possibilities are

$$
\begin{aligned}
& M_{1}=\left(\begin{array}{ccc}
0 & 0 & 0 \\
0 & 1 & 0 \\
0 & 0 & 1
\end{array}\right), \quad M_{2}=\left(\begin{array}{ccc}
0 & 0 & 1 \\
0 & 0 & 0 \\
1 & 0 & 1
\end{array}\right), \quad A \simeq \frac{k[x, y, z]}{\left(x^{2}, x y, y z, y^{2}-z^{2}\right)+(x, y, z)^{3}} \\
& M_{1}=\left(\begin{array}{lll}
0 & 0 & 0 \\
0 & 1 & 0 \\
0 & 0 & 1
\end{array}\right), \quad M_{2}=\left(\begin{array}{ccc}
0 & 0 & 1 \\
0 & 0 & 0 \\
1 & 0 & 1
\end{array}\right), \quad A \simeq \frac{k[x, y, z]}{\left(x^{2}, x y, y z, x z+y^{2}-z^{2}\right)+(x, y, z)^{3}} .
\end{aligned}
$$

If char $k \neq 2$, then the substitution $z \mapsto z-x / 2$ transforms the first into the second. If char $k=2$, then these two algebras are distinguished by the dimension of the kernel of the squaring map on $\mathfrak{m}$.

Case 4c: $a=0$ and $d \neq 0$. By symmetry in $x$ and $y$, this is the same as Case $4 \mathrm{~b}$.

Case 4d: $a=d=0$. Since $\operatorname{dim} \operatorname{ker}_{1}=\operatorname{dim} \operatorname{ker}_{2}=1$, we have $b, e \neq 0$. We may scale to assume that $b=e=1$, so we obtain

$$
M_{1}=\left(\begin{array}{lll}
0 & 0 & 0 \\
0 & 0 & 1 \\
0 & 1 & c
\end{array}\right), \quad M_{2}=\left(\begin{array}{ccc}
0 & 0 & 1 \\
0 & 0 & 0 \\
1 & 0 & f
\end{array}\right), \quad A \simeq \frac{k[x, y, z]}{\left(x^{2}, x y, y^{2}, z^{2}-(f x+c y) z\right)+(x, y, z)^{3}} .
$$

If char $k \neq 2$, a change of variable $z \mapsto z+(f / 2) x+(c / 2) y$ shows that

$$
A \simeq \frac{k[x, y, z]}{\left(x^{2}, x y, y^{2}, z^{2}\right)+(x, y, z)^{3}} .
$$

If char $k=2$, we can perform a linear change of the variables $x$ and $y$ to transform the linear form $f x+c y$ to either 0 or $x$; hence in addition to the algebra (1.7) we have the possibility

$$
A \simeq \frac{k[x, y, z]}{\left(x^{2}, x y, y^{2}, z^{2}-x z\right)+(x, y, z)^{3}},
$$

and this is not isomorphic to 1.7 , since only for 1.7$)$ does every element of $\mathfrak{m}$ have square 0 .

The algebras in Case 1 are distinguished from the others with $\vec{d}=(3,2)$ in that $F(t, u)$ is a nonzero cube, and are distinguished from the others in Case 1 by the equivalence type of the singular symmetric bilinear form. The two algebras in Case 2 are distinguished from the others in that the space $(A n n \mathfrak{m}) / \mathfrak{m}^{2}=\operatorname{ker}_{1} \cap \mathrm{ker}_{2}$ is nonzero, and are distinguished from each other by the isomorphism type of the symmetric form from $\mathfrak{m} /($ Ann $\mathfrak{m})$ to $\mathfrak{m}^{2}$ induced by multiplication. The remaining algebras, in Cases $3,4 \mathrm{a}, 4 \mathrm{~b}, 4 \mathrm{~d}$, have $F(t, u)$ equal to $-t^{2} u, t u(t+u),-t u^{2}, 0$, respectively. Therefore the only possible overlap is between Cases 3 and $4 \mathrm{~b}$. In both Case 3 and Case $4 \mathrm{~b}$, there are exactly two nonzero linear combinations of $\langle,\rangle_{1},\langle,\rangle_{2}$ up to scalar with a nonzero kernel, but only in Case 3 does one of them have a 2-dimensional kernel.

In all cases where we included $(x, y, z)^{3}$ in the ideal, we compute which monomials in $(x, y, z)^{3}$ are already in the ideal generated by the other generators, and include only the remaining ones when we describe the algebra in Table 1. This completes the determination of local algebras of rank $\leq 6$ over $k$.

Remark 1.8. If we fix $n \leq 6$ and $k$, there is a partial ordering on the set of isomorphism types of rank $n k$-algebras that expresses which types deform to which other types. It might be 
nice to give the Hasse diagram for this partially ordered set. This is done for $n=5$ with char $k \neq 2,3$ in [Maz80, p. 292].

Remark 1.9. Let $\mathfrak{B}_{n, k}$ be the moduli space of rank $n$ algebras equipped with a basis: see [Poo08]. There is an action of $\mathrm{GL}_{n}(k)$ on $\mathfrak{B}_{n, k}(k)$, and the orbits correspond to the isomorphism types of algebras. It follows that $\operatorname{dim} \mathfrak{B}_{n, k}=n^{2}$ for $n \leq 6$.

\section{Algebras of Rank at least 7}

Proposition 2.1 (|Sup56]). If $n \geq 7$ and $k$ is any infinite field, then the set of isomorphism classes of rank $n$ algebras over $k$ is infinite.

Proof. It suffices to consider $n=7$, because for higher $n$, we can take a product of a variable rank 7 algebra with $\prod_{i=8}^{n} k$. We analyze algebras $A$ with $\vec{d}=(4,2)$ as we did for $(3,2)$ above. They can be described by a linearly independent pair $\left(M_{1}, M_{2}\right)$ of symmetric $4 \times 4$ matrices, and the binary quartic form $\operatorname{det}\left(t M_{1}+u M_{2}\right)$ up to $\mathrm{GL}_{2}$-equivalence is determined by the isomorphism type of $A$. Considering the case where $M_{1}$ and $M_{2}$ are diagonal shows that all squarefree binary quartics arise. Now, given a squarefree binary quartic, we may choose an ordering of its zeros in $\mathbb{P}^{1}(\bar{k})$ and take their cross ratio. Since all cross ratios other than $0,1, \infty$ arise, but at most 4 ! (in fact, at most 6 ) come from each isomorphism class of algebras, there must be infinitely many algebras.

Remark 2.2. For an infinite field $k$ with char $k \neq 2$, we could give the following alternative argument. When char $k \neq 2$, symmetric matrices correspond to quadratic forms, so an algebra $A$ with $\vec{d}=(4,2)$ gives a pair of quadratic forms. Their common zero set in $\mathbb{P}^{3}$ defines in the generic case a smooth genus 1 curve, whose $j$-invariant is an invariant of $A$. Infinitely many $j$-invariants arise, so there are infinitely many isomorphism classes of $A$.

Remark 2.3. As pointed out by the referee, a similar argument can be used to simplify the case $\vec{d}=(3,2)$ handled in Section 1.5 , when char $k \neq 2$ : instead of a genus 1 curve, one obtains an intersection of two conics in $\mathbb{P}^{2}$, which generically is a 0 -dimensional scheme of degree 4 .

\section{Summary}

Table 2 lists, for each fixed rank $n$, the number of isomorphism types of rank $n$ local algebras over $k$, and the number of isomorphism types of all rank $n$ algebras; the numbers depend on char $k$. The counts for local algebras for char $k \neq 2,3$ agree with those given implicitly in Maz80].

\section{ACKNOWLEDGEMENTS}

I thank Marco Pellegrini, Bernd Sturmfels, Chiara Tamburini, and Bianca Viray for corrections, and Gianfranco Casnati and Alice Fialowski for comments. This work was done mainly during the workshop on "Rings of low rank" at the Lorentz Center in 2006. Some of the calculations were checked using the Magma computational algebra system. The research was partially supported by NSF grant DMS-0301280. 


\begin{tabular}{c|ccc|ccc} 
& \multicolumn{3}{|c|}{ Local } & \multicolumn{3}{c}{ All } \\
$n$ & char $\neq 2,3$ & char 2 & char 3 & char $\neq 2,3$ & char 2 & char 3 \\
\hline 0 & 0 & 0 & 0 & 1 & 1 & 1 \\
1 & 1 & 1 & 1 & 1 & 1 & 1 \\
2 & 1 & 1 & 1 & 2 & 2 & 2 \\
3 & 2 & 2 & 2 & 4 & 4 & 4 \\
4 & 4 & 5 & 4 & 9 & 10 & 9 \\
5 & 9 & 10 & 9 & 20 & 23 & 20 \\
6 & 25 & 33 & 26 & 53 & 64 & 54 \\
$\geq 7$ & $\infty$ & $\infty$ & $\infty$ & $\infty$ & $\infty$ & $\infty$
\end{tabular}

TABLE 2. Number of rank $n$ algebras over $k=\bar{k}$ up to isomorphism.

\section{REFERENCES}

[Alb38] A. Adrian Albert, Symmetric and alternate matrices in an arbitrary field. I, Trans. Amer. Math. Soc. 43 (1938), no. 3, 386-436, DOI 10.2307/1990068. MR1501952 \1.4

[Bha04a] Manjul Bhargava, Higher composition laws. I. A new view on Gauss composition, and quadratic generalizations, Ann. of Math. (2) 159 (2004), no. 1, 217-250. MR2051392 (2005f:11062a) 11

[Bha04b] , Higher composition laws. II. On cubic analogues of Gauss composition, Ann. of Math. (2) 159 (2004), no. 2, 865-886. MR2081442 (2005f:11062b) 11

[Bha04c] _ Higher composition laws. III. The parametrization of quartic rings, Ann. of Math. (2) 159 (2004), no. 3, 1329-1360. MR2113024 (2005k:11214) 11

[Bha05] _ The density of discriminants of quartic rings and fields, Ann. of Math. (2) 162 (2005), no. 2, 1031-1063. MR2183288 11

[Cha54] B. Charles, Sur l'algèbre des opérateurs linéaires, J. Maths. Pures. Appl. (9) 33 (1954), 81-145 (French). MR0065521 (16,439a) 11.1

[Dym66] Z. M. Dyment, Maximal commutative nilpotent subalgebras of a matrix algebra of the sixth degree, Vesci Akad. Navuk BSSR Ser. Fiz.-Mat. Navuk 1966 (1966), no. 3, 53-68 (Russian). MR0202762 (34 \#2622) 11.1

[Maz80] Guerino Mazzola, Generic finite schemes and Hochschild cocycles, Comment. Math. Helv. 55 (1980), no. 2, 267-293. MR576606 (82k:14010) 11.1, 1.8, 3

[PTB16] M.A. Pellegrini and M.C. Tamburini Bellani, More on regular subgroups of the affine group, January 7, 2016. Preprint, arXiv:1601.01679v1 . 11.2

[Poo08] Bjorn Poonen, The moduli space of commutative algebras of finite rank, J. Eur. Math. Soc. (JEMS) 10 (2008), no. 3, 817-836, DOI 10.4171/JEMS/131. MR2421162 (2009d:14009) 11, 1.9

[Sup56] D. A. Suprunenko, On maximal commutative subalgebras of the full linear algebra, Uspehi Mat. Nauk (N.S.) 11 (1956), no. 3(69), 181-184 (Russian). MR0082983 (18,639h) 11. 2.1

[ST66] D. A. Suprunenko and R. I. Tyškevič, Perestanovochnye matritsy, Nauka i Tehnika, Minsk, 1966 (Russian); English transl. in Commutative matrices, Academic Press, 1968. MR0201472 (34 \#1356) 1.1

Department of Mathematics, University of California, Berkeley, CA 94720-3840, USA

Current address: Department of Mathematics, Massachusetts Institute of Technology, Cambridge, MA 02139-4307, USA

Email address: poonen@math.mit.edu

$U R L:$ http://math.mit.edu/ poonen/ 